\title{
OPEN Detection of selection signatures for response to Aleutian mink disease virus infection in American mink
}

\author{
Karim Karimi ${ }^{1}$, A. Hossain Farid $^{1}$, Sean Myles ${ }^{2}$ \& Younes Miar ${ }^{1 凶}$
}

Aleutian disease (AD) is the most significant health issue for farmed American mink. The objective of this study was to identify the genomic regions subjected to selection for response to infection with Aleutian mink disease virus (AMDV) in American mink using genotyping by sequencing (GBS) data. A total of 225 black mink were inoculated with AMDV and genotyped using a GBS assay based on the sequencing of ApeKI-digested libraries. Five AD-characterized phenotypes were used to assign animals to pairwise groups. Signatures of selection were detected using integrated measurement of fixation index $\left(F_{S T}\right)$ and nucleotide diversity $(\theta \pi)$, that were validated by haplotype-based (hap-FLK) test. The total of 99 putatively selected regions harbouring 63 genes were detected in different groups. The gene ontology revealed numerous genes related to immune response (e.g. TRAF3IP2, WDR7, SWAP70, CBFB, and GPR65), liver development (e.g. SULF2, SRSF5) and reproduction process (e.g. FBXO5, CatSperB, CATSPER4, and IGF2R). The hapFLK test supported two strongly selected regions that contained five candidate genes related to immune response, virus-host interaction, reproduction and liver regeneration. This study provided the first map of putative selection signals of response to AMDV infection in American mink, bringing new insights into genomic regions controlling the AD phenotypes.

Aleutian disease (AD), caused by a contagious parvovirus in the genus Amdoparvovirus, is the most significant health issue for farmed American mink (Neovison vison) across the world. Infection with Aleutian mink disease virus (AMDV) stimulates immune responses to produce high levels of persistent antibodies which cannot neutralize the virus, and subsequently leads to the formation and deposition of immune complexes causing kidney damage and high mortality especially in kits ${ }^{1}$. In addition, one or more of other symptoms such as arteritis ${ }^{2}$, hepatitis ${ }^{3}$ and splenomegaly ${ }^{4}$ were reported in some infected adult mink. Furthermore, AD is generally associated with infertility, abortion, low fur quality and weight loss of infected animals ${ }^{5,6}$, leading to prominent economic losses in mink industry worldwide.

Although immunological tests have been used to detect and eliminate infected animals, this strategy has not been successful in complete eradication of the virus from mink farms. For instance, despite the successful use of counterimmunoelectrophoresis (CIEP) test to reduce the prevalence of infection on mink farms in the Canadian province of Nova Scotia, this strategy failed to eradicate the virus on most farms ${ }^{7}$. In addition, a high persistence of AMDV was observed in the breeding environments causing the reinfection of mink farms and making it difficult to control the prevalence of the disease ${ }^{8,9}$. Thus far, no effective vaccine or treatment is available for $\mathrm{AD}^{10}$. However, some non-Aleutian mink can tolerate the infection, showing normal levels of serum gamma-globulin, low anti-AMDV antibody titer, and mild or no gross or microscopic lesions characteristic of $\mathrm{AD}^{11-14}$. Moreover, it is well documented that some AMDV-infected mink do not succumb to the disease and live healthy lives ${ }^{14-16}$, suggesting that selection for tolerance to $\mathrm{AD}$ is a feasible approach to control $\mathrm{AD}$ on mink farms. Characterizing the genomic regions contributing to $\mathrm{AD}$ tolerance could be an effective way to develop breeding programs for increased $\mathrm{AD}$ resiliency on mink farms.

Development of whole-genome sequencing technologies has facilitated the identification of single nucleotide polymorphisms (SNPs) across the genome and provided a powerful tool to discover the genomic regions targeted by selection pressures in different species ${ }^{17}$. Genome-wide detection of selection signatures has widely been used to understand the genetic basis of domestication ${ }^{18,19}$, the process of adaptation to extreme environments ${ }^{20,21}$, and 


\begin{tabular}{|c|c|c|c|c|c|}
\hline \multirow[b]{2}{*}{ Group } & \multirow[b]{2}{*}{ Measurements } & \multicolumn{2}{|l|}{ Positive subgroup } & \multicolumn{2}{|l|}{ Negative subgroup } \\
\hline & & Number of individuals & Description & Number of individuals & Description \\
\hline Survival & Survived or death due to sickness & 20 & $\begin{array}{l}\text { Mink which died or terminated } \\
\text { due to sickness and were PCR } \\
\text { positive at day } 35 \text { pi }\end{array}$ & 52 & $\begin{array}{l}\text { Mink were alive until euthanasia } \\
\text { and had no lesions in the lungs, } \\
\text { liver and kidneys }\end{array}$ \\
\hline Kidney lesions & $\begin{array}{l}\text { Severity of AD symptoms in } \\
\text { kidneys }\end{array}$ & 30 & $\begin{array}{l}\text { Inoculated mink had lesions ( } 1 \text { to } \\
4 \text { scales of severity) }\end{array}$ & 142 & Mink had no lesion in kidneys \\
\hline Antibody titer & $\begin{array}{l}\text { CIEP test of serially diluted } \\
\text { plasma }\end{array}$ & 23 & Antibody titres $\leq 1 / 128$ & 56 & Antibody titres $\geq 1 / 4$ \\
\hline Virus clearance & Virus in the spleen & 128 & Positive PCR test & 43 & Negative PCR test \\
\hline Viremia-350 & Virus in plasma, day $350 \mathrm{pi}$ & 17 & Positive PCR test & 156 & Negative PCR test \\
\hline
\end{tabular}

Table 1. The phenotypic traits used to classify individuals in five pairwise groups based on the response to AMDV infection.

the effects of positive selection on genetic composition of economically important traits in livestock species ${ }^{22,23}$. However, to our knowledge, no study has been conducted in American mink to scan the genome for selective sweeps. Identification of selection signatures in American mink requires genotyping a huge number of SNP markers throughout the genome in many individuals. Genotyping by sequencing (GBS) is a cost-effective approach to discover a large number of SNPs across the genome using next generation sequencing technologies ${ }^{24}$. The use of this approach can provide the opportunity to identify DNA markers underlying the adaptive changes for the traits of interest in American mink.

The objective of this study was to use the genome-wide SNP markers extracted by GBS technique to reveal the genomic regions under selection in a group of black American mink inoculated with AMDV.

\section{Materials and methods}

Animals and sampling. Animal management and sampling protocols were performed in accordance with the standards of the Canadian Council on Animal Care (http://www.ccac.ca) after approval by the Dalhousie University Animal Care and Use Committee. Prior to inoculation, sampling and euthanasia, animals were anesthetized as previously explained ${ }^{25}$. Animals which were used in the current experiment were chosen from a group of 1435 black American mink which were inoculated in the Falls of 2010, 2011 and 2012. The viral inoculum was a $10 \%(\mathrm{~W} / \mathrm{V})$ passage 2 of AMDV prepared from the spleens of mink infected with a local strain and stored at $-80^{\circ} \mathrm{C}$ as previously described ${ }^{25}$. Animals were intranasally inoculated under sedation with $60 \mu \mathrm{L}$ of the viral homogenate, which corresponded to approximately 300 to $700 \mathrm{ID}_{50}$, depending on the method and time of detection of infection ${ }^{26}$. Intranasal inoculation of sedated mink was found to be an effective method of establishing infection without destroying animals physical barrier ${ }^{27}$, thus resembling natural infection. Animals were kept in individual cages at a bio-secure facility (Aleutian Disease Research Centre) and fed a commercial dry pellet (National Feeds Inc., Maria Stern, OH, USA, https://www.manta.com/c/mr55j1c/national-feeds-inc). Animals had free access to feed and water and were monitored daily. The date and signs of dead and sick animals were recorded. Healthy animals with high reproductive performance were retained for breeding, and extra animals were killed in January and February each year.

Blood samples were collected by toenail clipping under anesthesia at least three times each year, starting at day 35 post-inoculation (pi). Blood was collected in heparinized capillary tubes for antibody testing by the CIEP, and in EDTA-coated capillary tubes for viral DNA detection by the polymerase chain reaction (PCR). By the time of conducting this experiment, 644 of the mink had been euthanized and their blood, lungs, liver, kidneys, heart and spleen samples had been collected. The lungs, kidneys and liver samples were stored in $10 \%$ formalin for histopathological evaluation. Among the inoculated animals, 392 individuals had died or terminated due to AD.

Laboratory procedures. The presence of antibodies in the plasma was evaluated using CIEP test ${ }^{12}$, and antibody titers of euthanized mink were measured by CIEP using 11 two-fold serial dilutions of plasma samples $(1,1 / 2,1 / 4,1 / 8,1 / 16,1 / 32,1 / 64,1 / 128,1 / 256,1 / 512$ and 1/1024). Antibody titer was recorded as the highest level of dilution that resulted in positive CIEP test ${ }^{25}$. DNA was extracted from plasma and from cell-free tissue suspensions using Dynabeads Silane viral nucleic acid extraction kit (Invitrogen, Burlington, ON). The PCR was used to test the presence of viral DNA using primer $60 \mathrm{~F}$ and $60 \mathrm{R}$ as previously explained ${ }^{28}$. Furthermore, severity of AD symptoms was determined in the lung, kidney and liver samples by an experienced pathologist at the Pathology Laboratory, Nova Scotia Department of Agriculture (Truro, Canada). Histolopathological lesions were measured based on a scale from 0 (no lesion) to 4 (very severe lesions), as an indicator of accumulation of plasma cells in tissues ${ }^{29}$.

Libraries preparation and DNA sequencing. The mink that were selected for this experiment $(n=225)$ were those with a wide range of responses to AMDV infection (Table 1). High quality DNA was extracted from the spleen tissue of each animal using the high-salt method ${ }^{30}$. Quality of DNA was checked by gel electrophoresis and DNA samples were quantified by a NanoDrop 1000 spectrophotometer (Thermo Scientific, Waltham, MA, USA). Samples with 260/280 ratio greater than 1.7 were selected to assure purity of DNA. High quality DNA was digested with the restriction enzyme ApeKI. Barcode adaptors along with a standard Y-adaptor were ligated 
to DNA fragments. DNA amplification was performed by PCR to generate three sets of 96-plex GBS libraries. Finally, DNA sequencing was performed on three lanes of the Illumina HiSeq Sequencer at the Génome Québec Innovation Centre.

Sequences analysis and quality control. In total, more than 681 million of $100 \mathrm{bp}$ single-end reads were generated by the Illumina HiSeq Sequencer. Unique adaptor barcodes were used to demultiplex reads into separate files using Sabre software (https://github.com/najoshi/sabre). Primers sequences, adaptor contaminations and all reads shorter than $50 \mathrm{bp}$ were discarded using Cutadapt $\mathrm{t}^{31}$. All reads were mapped to the American mink reference genome $e^{32}$ using BWA-MEM with default parameters ${ }^{33}$. Then, the variant calling was performed by Genome Analysis ToolKit (GATK)'s HaplotypeCaller ${ }^{34}$. The following metrics were implemented in GATK to filter variants with a quality by depth $(\mathrm{QD})<2.0$, mapping quality $(\mathrm{MQ})<40.0$, Fisher strand $(\mathrm{FS})>60.0$, mapping quality rank sum test $<-12.5$, and read position rank sum test $<-8.0$. Furthermore, all SNPs with a minor allele frequency $(\mathrm{MAF})<0.05$, call rate $<0.90$ and those deviating from Hardy-Weinberg equilibrium $\left(P<10^{-6}\right)$ were filtered out. In addition, individuals with $>0.15$ missing genotypes were discarded from the data set using VCFtools ${ }^{35}$. Minor allele frequency was computed for all SNPs and proportion of SNPs was determined for MAF ranges of $<0.05,0.05$ to $<0.1,0.1$ to $<0.2,0.2$ to $<0.3,0.3$ to $<0.4$ and 0.4 to $\leq 0.5$.

Animals grouping. Five phenotypic parameters including antibody titer, survival or death due to AD, severity of $\mathrm{AD}$ symptoms in the kidneys, virus clearance (the presence or absence of AMDV DNA in the spleen) and viremia on day 350 pi (PCR on plasma) were used to divide animals into positive and negative subgroups based on the level of response to AMDV infection. Table 1 presents the five pairwise groups as well as the number of individuals in each subgroup and the criteria considered for each grouping. In addition, Supplementary Fig. S1 shows the number of individuals shared among different phenotypic groups.

Detection of selection signatures. Analysis using genetic differentiation measures. In this study, several approaches were used to detect selection signatures in the genome of American mink. First, a genome scan for selection signatures was performed based on the combination of genome-wide pairwise $\mathrm{F}_{\mathrm{ST}}^{36}$ and nucleotide diversity $\left(\theta_{\pi}\right)^{37}$ within each group of animals. Both statistics were calculated for each SNP and averaged along $100 \mathrm{~kb}$ windows with a step size of $25 \mathrm{~kb}$ using VCFtools. The $\mathrm{F}_{\mathrm{ST}}$ values were then $\mathrm{Z}$-transformed $\left(\mathrm{Z}\left(\mathrm{F}_{\mathrm{ST}}\right)\right)$ and visualised using a scaffold-based circos plot $^{38}$ for all groups. Moreover, $\theta_{\pi}$ ratios were computed as $\theta_{\pi \text {-Negative }}$ $\theta_{\pi \text {-Positive }}$ for all pairs of groups and were then $\log _{2}$-transformed $\left(\log _{2}\left(\theta_{\pi \text { ratios }}\right)\right)$. All of the windows including the top $1 \%$ values of both $\mathrm{Z}\left(\mathrm{F}_{\mathrm{ST}}\right)$ and $\log _{2}\left(\theta_{\pi \text { ratios }}\right)$ were considered as the candidate selection regions. Since the results of differentiation methods are likely to be inaccurate for small scaffolds, only scaffolds $\geq 10 \mathrm{Mb}$ (134 scaffolds including 29,914 SNPs) were used in the analyses.

Analysis using hapFLK test. The hapFLK test uses the local haplotype structures as well as haplotype allele frequencies and hierarchical structure of populations to reveal the signatures of selection. This approach takes into account a neutral model for SNP data and can be applied to unphased genomic data ${ }^{39}$. The hapFLK test was used to further validate the candidate regions identified by the previously described measures. We used the haplotype-based statistic (hapFLK) to reveal the selection signals accounting for differentiation of haplotype structures among groups. Various numbers of haplotype clusters (- K 10 to - K 40) were tested using fastPHASE software $^{40}$ and $-\mathrm{K} 30$ was selected as the best number of clusters in the hapFLK analyses. The kinship matrix and Reynolds' genetic distance were computed by hapFLK v.1.40 for each scaffold. In addition, no outgroup was considered, and the expected maximum number of iterations was set to be 20 to fit the LD model.

P-value calculation. It was assumed that the putatively selected regions include only a small fraction of the genome ${ }^{41}$. Therefore, the distribution of hapFLK values can be explained by a normal distribution except for a small proportion of outliers related to selected regions. The mean and variation of the hapFLK values were estimated using MASS package in R. The hapFLK statistics were then Z-transformed using these parameters and $P$-values were computed assuming a normal distribution in R. False discovery rate (FDR) approach ${ }^{42}$ was then performed using $\mathrm{q}$-value package in $\mathrm{R}$ to correct $P$-values for multiple testing. The regions including $\mathrm{q}$-value $<0.05$ were considered as potentially selected regions.

Gene ontology and functional analysis. We used the biomaRt package of R to find all Ensembl gene IDs overlapped with the candidate regions. Since the domestic dog (Canis lupus familiaris) is known as the closest species to mink whose genome has widely been annotated ${ }^{32}$, the gene ontology was conducted based on the reference list of this species. The biological process, molecular function and cellular component terms were assessed for all genes using PANTHER 14.1 ${ }^{43}$. Statistical overrepresentation of annotated genes was assessed by Fisher's exact test and corrected by false discovery rate (FDR) procedure. These genes were further investigated by reviewing relevant literatures in relation to the phenotypes or pathways of interest in different groups.

\section{Results}

Data quality control. The total of $681,936,405$ reads was generated with an average of 2,133,590 reads per sample. Overall, $97.12 \%$ of reads were mapped to the mink genome assembly with a range of $91.14 \%$ to $97.59 \%$ among samples. A data set including 62,404 SNPs across 1,834 scaffolds was produced after variant calling. These scaffolds covered $1.75 \mathrm{~Gb}$ of the genome, which captured approximately $73 \%$ of the $2.4 \mathrm{~Gb}$ reference genome. After quality control, 47,800 SNPs from 216 animals were kept for further analyses. The average number of SNPs 


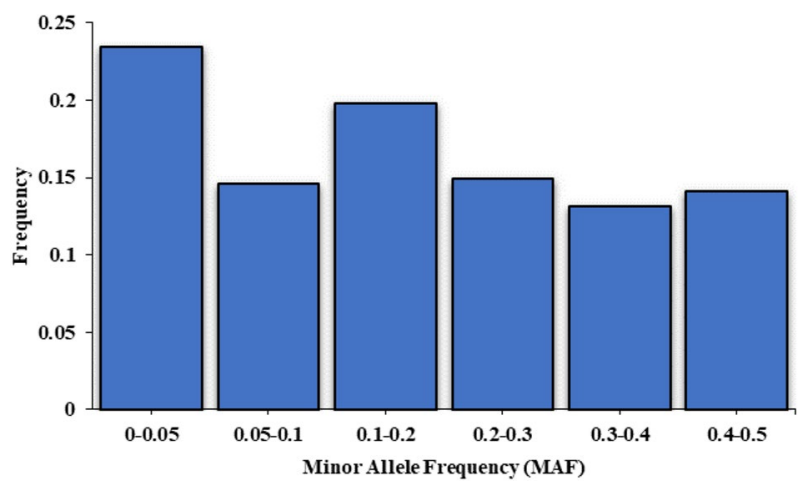

Figure 1. Distribution of minor allele frequencies (MAF) across the data set obtained by GBS in American mink.

\begin{tabular}{|l|l|l|}
\hline Group & Number of candidate regions identified & Number of genes \\
\hline Survival & 20 & 13 \\
\hline Kidney lesions & 34 & 21 \\
\hline Antibody titer & 12 & 8 \\
\hline Virus clearance & 21 & 11 \\
\hline Viremia-350 & 12 & 10 \\
\hline Total & 99 & 63 \\
\hline
\end{tabular}

Table 2. Number of candidate regions and genes detected by overlapping $Z\left(\mathrm{~F}_{\mathrm{ST}}\right)$ and $\log _{2}\left(\theta_{\pi \text { ratios }}\right)$ in five differential groups of responses to AMDV-infection. Total do not consider overlap between groups.

per scaffold was 34 with the maximum number of 1,320 SNPs on scaffold 10. The average MAF was $0.19 \pm 0.15$ across all SNPs. Figure 1 presents the proportion of SNPs with different ranges of MAF. A large proportion of SNPs (23\%) had MAF $<0.05$ whereas the lowest number of SNPs was observed in the range of $0.3 \leq \mathrm{MAF}<0.4$ $(13 \%)$.

Genome-wide signatures of selection. Genetic differentiation statistics. We measured pooled $\mathrm{F}_{\mathrm{ST}}$ in $100 \mathrm{~kb}$ windows between opposing pairs of each group. The total of 28,305 windows with step sizes of $25 \mathrm{~kb}$ was scanned along the genome with the average number of $4.6 \pm 7.3 \mathrm{SNPs}$ per $100 \mathrm{~kb}$ window. Supplementary Fig. S2 presents the distribution of $\mathrm{F}_{\mathrm{ST}}$ on the scaffolds showing potential signatures of selection in different groups. Each phenotypic group showed some contiguous windows including high levels of $\mathrm{F}_{\mathrm{ST}}$, which were potentially the candidate regions of positive selection. A complete list of the top $1 \%$ of $\mathrm{Z}\left(\mathrm{F}_{\mathrm{ST}}\right)$ distribution was provided for each group in the Supplementary Dataset S1. The differentiation of individuals within each group was also assessed based on $\theta_{\pi}$ ratios (i.e. $\theta_{\pi \text {-Negative }} / \theta_{\pi \text {-Positive }}$ ). Supplementary Fig. S3-S7 present the overlap of top $1 \%$ values between $\mathrm{Z}\left(\mathrm{F}_{\mathrm{ST}}\right)$ and $\log _{2}\left(\theta_{\pi \text { ratios }}\right)$ in different groups. In addition, a complete list of candidate regions along with their positions was provided in Supplementary Table S1. Total number of identified candidate regions was 99 and varied from 12 for antibody titer and viremia-350 groups to 34 for kidney lesion scores (Table 2). These regions harboured 63 genes potentially subjected to selection for response to AMDV infection (Supplementary Table S2). A total of seven candidate regions were shared between at least two phenotypic groups (Table 3). The group with the most shared candidate regions was survival (4 regions). On the other hand, viremia-350 shared only two regions with survival and antibody titer groups.

Figure 2 presents the pie chart of molecular functions attributed to candidate genes in the putative regions of selection. These results indicated that a significant proportion of genes were involved in binding (38.3\%) and catalytic (31.9\%) activities. In addition, gene ontology analysis resulted in 109 overrepresented $(P<0.05)$ GO enrichment terms related to biological process (Supplementary Dataset S2). For instance, the candidate genes were highly enriched in Wnt signaling, calcium modulating (Wnt/Ca2+) pathways (GO:0007223), liver regeneration (GO:0097421), animal organ regeneration (GO:0031100), cardiac muscle cell proliferation (GO:0060038), response to isoquinoline alkaloid (GO:0014072), zinc ion transport (GO:0006829), response to progesterone (GO:0032570) and response to stimulus (GO:0050896), which were relevant to AD-characterized phenotypes (Supplementary Dataset S2). In addition, the gene ontology revealed the biological roles of several genes related to immune system process (TRAF3IP2, WDR7, TNFRSF11A, SWAP70, CBFB, IGF2R and GPR65), response to stress (SULF2, CADPS2, NOX3, GNAO1, HSF4, AMFR and CNOT1), reproduction (FBXO5, CatSper $\beta$, MAS1, CATSPER4, GOT2 and IGF2R) and regulation of nervous system development (TENM4, VSNL1, CLSTN1, RIT2, TCF4, SKOR2 and NDRG4). 


\begin{tabular}{|l|l|l|l|l|l|l|}
\hline Scaffolds & Position (bp) & Survival & Kidney lesions & Antibody titer & Virus clearance & Viremia-350 \\
\hline 1 & $23,000,001$ & & & $\times$ & $\times$ & \\
\hline 2 & $13,750,001$ & $\times$ & & $\times$ & & \\
\hline 2 & $23,425,001$ & $\times$ & & $\times$ & & $\times$ \\
\hline 2 & $27,325,001$ & $\times$ & & & & \\
\hline 5 & $13,850,001$ & $\times$ & $\times$ & & & \\
\hline 6 & $13,475,001$ & & $\times$ & & $\times$ & \\
\hline 6 & $18,375,001$ & & $\times$ & & $\times$ & \\
\hline
\end{tabular}

Table 3. Candidate regions (spans $\pm 1 \mathrm{Mb}$ ) identified by overlapping selective signals of $\mathrm{Z}\left(\mathrm{F}_{\mathrm{ST}}\right)$ and $\log _{2}$ $\left(\theta_{\pi \text { ratios }}\right)$ that were shared by two or more groups.

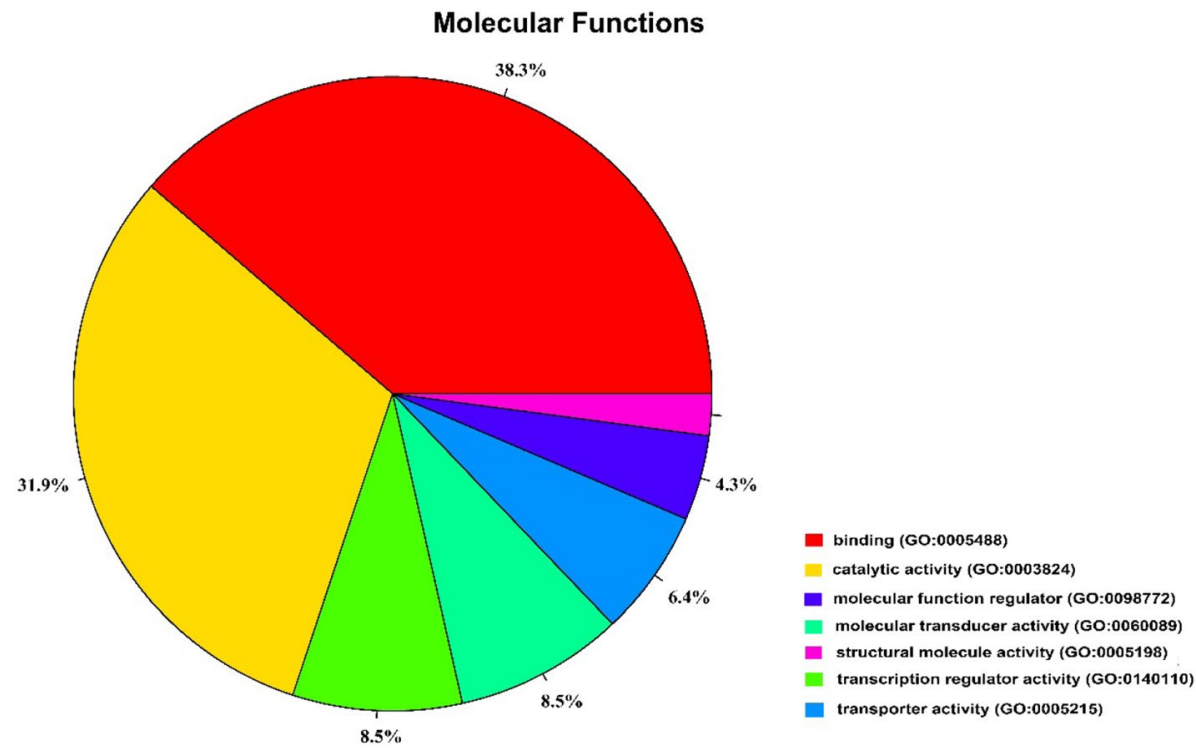

Figure 2. The pie chart of molecular functions attributed to candidate genes detected by overlapping selective signals of $\mathrm{Z}\left(\mathrm{F}_{\mathrm{ST}}\right)$ and $\log _{2}\left(\theta_{\pi \text { ratios }}\right)$.

HapFLK test. We used the hapFLK statistic to validate the signatures of selection detected by aforementioned methods. The hapFLK values were computed only for scaffolds containing significant candidate regions from previous approach. Figure 3 presents $-\log _{10}(q$-value) of hapFLK statistics per each genomic position in different groups. Table 4 presents the putative regions identified based on hapFLK test along with the candidate genes located in these regions. Among all ten candidate regions detected by hapFLK test, five regions were overlapped with those positions identified by previous approaches (Table 4). The GO enrichment analysis indicated that selected regions were involved in 71 biological process GO terms (Supplementary Dataset S2). A fold enrichment $>100$ was observed for some biological processes such as neutrophil degranulation (GO:0043312), liver regeneration (GO:0097421), neutrophil activation involved in immune response (GO:0002283), leukocyte degranulation (GO:0043299), androgen receptor signaling pathway (GO:0030521) and macrophage differentiation (GO:0030225).

\section{Discussion}

The SNP data set generated by GBS technique was used to scan the American mink genome for positions that might have been targeted by selection for response to AMDV infection. A large proportion of sequencing reads (on average, 97.12\%) were mapped to the mink genome reference, which revealed the efficiency of methodology applied in this study. The GBS approach was previously used to identify markers associated with body size and pelt length in American mink ${ }^{44}$. The number of detected SNP markers in the current study $(62,404)$ is higher than that $(34,816)$ reported by Cai et al. ${ }^{44}$, that might mainly be attributed to different restriction enzymes applied to digest DNA sequences in these studies. Although, similar to the results by Cai et al. ${ }^{44}$, high percentage (23\%) of SNPs had MAF $<0.05$, the uniformity of MAF distribution was higher in the current study (Fig. 1). In addition, the GBS method has previously been used to identify the signatures of selection in other domestic animals e.g. Duroc pigs ${ }^{45}$ and Dromedary camels ${ }^{46}$. Our results suggested that GBS technique is an efficient approach to perform genome-wide studies in American mink, where there is no SNP panel available so far. 
a)

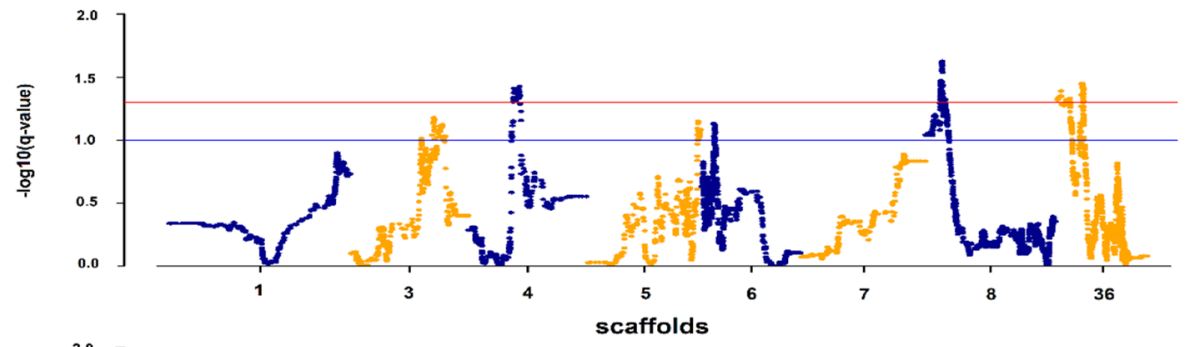

b)

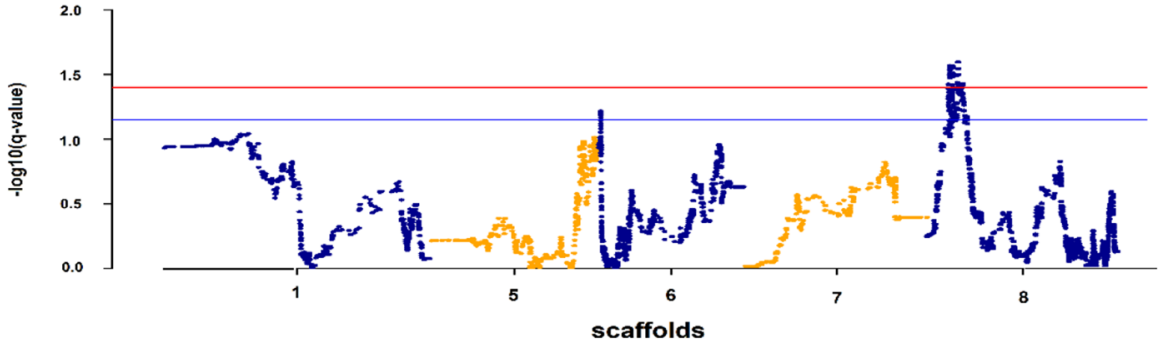

c)

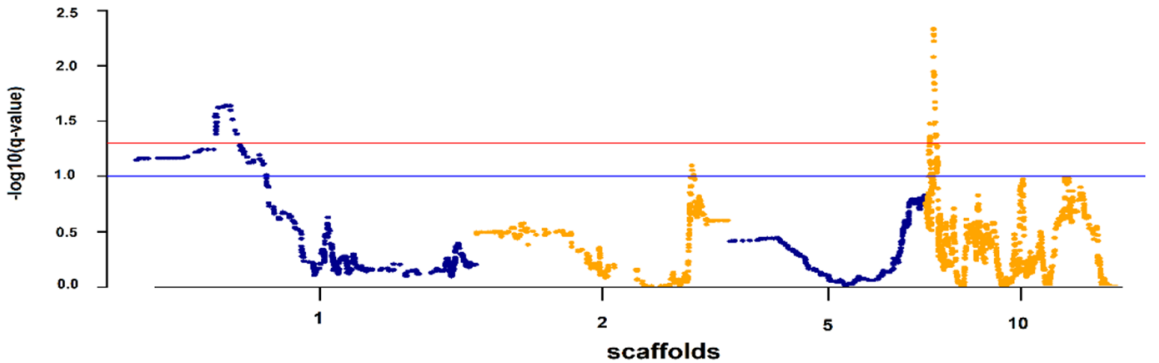

d)

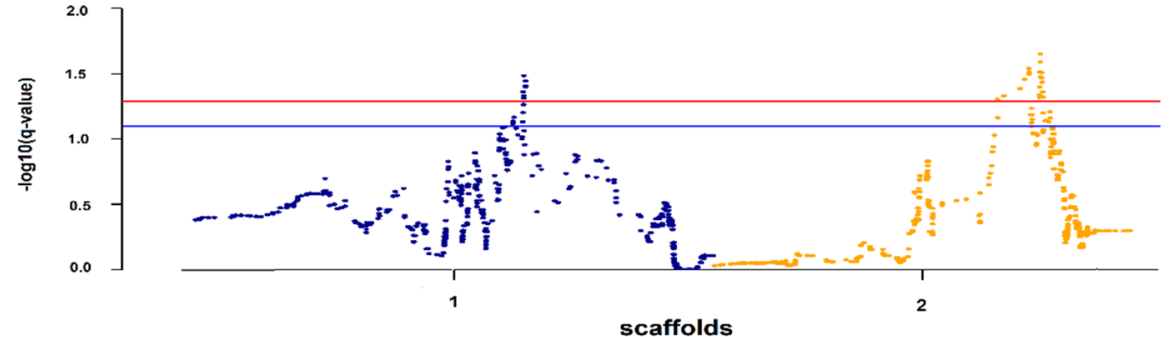

e)

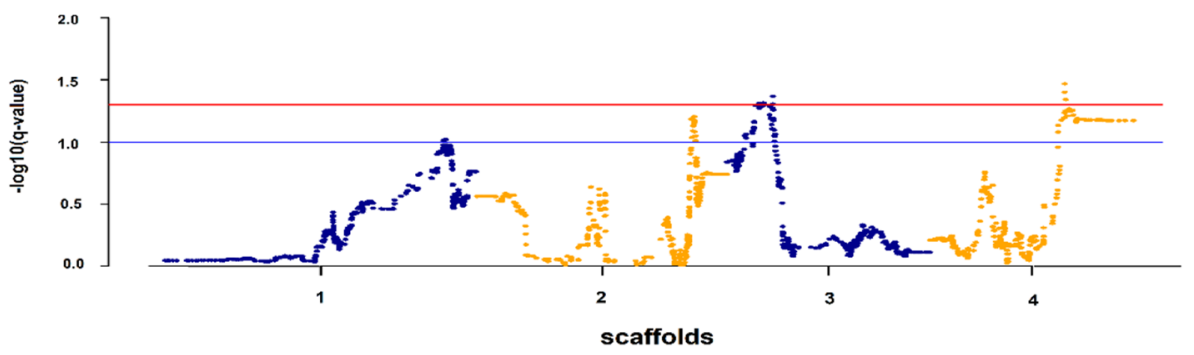

Figure 3. Manhattan plots of hapFLK statistic in different groups: (a) kidney lesions (b) virus clearance (c) survival $(\mathbf{d})$ antibody titer and $(\mathbf{e})$ viremia-350. Blue and red lines indicate suggestive $(\mathrm{q}$-value $<0.10)$ and significant (q-value $<0.05)$ thresholds, respectively.

In the current study, the phenotypic variation within the population was used to classify the animals to different subpopulations. Use of inter-population selection signatures approach can be exemplified by the studies of litter size trait in dairy goat ${ }^{47}$, backfat thickness in Yorkshire pigs ${ }^{48}$, adaptation to different environments in Atlantic salmon ${ }^{49}$ and disease risk in Japanese people ${ }^{50}$. The current study revealed several loci which have likely been under selection by combining $\mathrm{F}_{\mathrm{ST}}$ and $\theta \pi$ scores, and validated by haplotype-based (hap-FLK) tests. Given the fact that it was difficult to determine a strict threshold to distinguish homozygous regions undergoing selection from those caused by genetic drift, we combined the top $1 \%$ genomic regions obtained by both $\mathrm{F}_{\mathrm{ST}}$ and $\theta \pi$ to narrow our results. In addition, since the unknown demography of the population made it difficult to interpret the distribution of statistics under the null hypothesis of no selection and weakened the detection of signatures of selection $^{51}$, we integrated the statistical approaches to increase the likelihood of pinpointing to genomic regions that most likely contributed to true signatures of selection within the population. 


\begin{tabular}{|c|c|c|c|c|c|}
\hline Scaffolds & Position (bp) & Group & Lowest q-value & Gene symbols & Overlapping status $^{\mathrm{a}}$ \\
\hline 1 & $9,129,632-9,979,233$ & Survival & 0.023 & BMP4, CDKN3, CNIH1, GMFB, CGRRF1, SAMD4A & - \\
\hline 10 & $915,739-1,126,382$ & Survival & 0.004 & AJAP1 & - \\
\hline 1 & $23,046,774-23,187,210$ & Antibody titer & 0.034 & SRSF5, SLC10A1 & $\times$ \\
\hline 2 & $23,424,424-23,969,935$ & Antibody titer & 0.022 & RNF165, LOXHD1, SKOR2 & $\times$ \\
\hline 3 & $4,297,807-4,310,786$ & Viremia-350 & 0.048 & $W W P 2$ & - \\
\hline 4 & $16,076,915-16,196,438$ & Viremia-350 & 0.034 & PTPRZ1 & - \\
\hline 4 & $10,514,110-10,841,037$ & Kidney lesions & 0.037 & KLHDC10, ZC3HC1, UBE2H & $x$ \\
\hline 8 & $3,478,058-4,850,122$ & Kidney lesions & 0.023 & SULF2, EYA2, SLC2A10, TP53RK, SLC35C2 & $x$ \\
\hline 36 & $5,250,514-5,725,445$ & Kidney lesions & 0.035 & ATE1, NSMCE4A, TACC2 & - \\
\hline 8 & $3,620,504-5,308,341$ & Virus clearance & 0.025 & $\begin{array}{l}\text { SULF2, EYA2, SLC2A10, TP53RK, SLC35C2, PLTP, MMP-9, CTSA, ELMO2, } \\
\text { OCSTAMP, UBE2C, ACOT8 }\end{array}$ & $x$ \\
\hline
\end{tabular}

Table 4. Candidate regions identified by hapFLK test along with genes involving in these regions. ${ }^{\mathrm{a}}$ Overlaps with those regions detected by integrated measurement of $\mathrm{F}_{\mathrm{ST}}$ and $\theta_{\pi}$.

Despite the existence of a large number of non-viremic samples at day 350 pi (156 individuals), most of these animals (128 individuals) harboured the virus (PCR positive spleen) when euthanized (Table 1). It is well established that AMDV replication peaks around day 10 pi and then declines ${ }^{1}$, which causes irregular and short-lived viremia in most mink ${ }^{13,26,27,52,53}$. The virus remains in the lymphoid organs, such as the spleen, which are the primary sites of virus replication and sequestration ${ }^{1}$, and can be detected in the lymphoid organs long after the drop in viral replication and the absence of viremia ${ }^{26}$. Only a small number of mink are able to clear the virus, as shown in the current and previous studies ${ }^{13,15,54}$.

A total of 99 candidate selection regions were identified on ten scaffolds by overlapping the top $1 \%$ of $Z\left(\mathrm{~F}_{\mathrm{ST}}\right)$ and $\log _{2}(\theta \pi$ ratios). These regions were annotated by 63 genes based on the Ensembl genome database. Gene ontology indicated that most of these genes (38.3\%) were enriched in binding activity $(\mathrm{GO}: 0,005,488)$, which can be attributed to the mechanisms involved in the disease progress. The AMDV infection causes an immune complex-mediated disease, which is characterized by hypergammaglobulinemia and leads to penetrate viral particles into target cells through virus-antibody complexes ${ }^{55,56}$. Therefore, reactions against developing AD might be associated with genes controlling binding activity. Furthermore, high enrichment of genes involved in catalytic activities might be related to wide histopathological reactions of liver and kidneys due to AD progression ${ }^{57}$. Furthermore, several biological process GO terms were found in connection with AD target phenotypes (Supplementary Dataset S2). For example, it was demonstrated that Wnt/Ca2 + signalling pathway plays a major role in inflammatory responses and co-operative activation of innate and adaptive immunity ${ }^{58}$.

Immunity responses. Since AD causes a virus-induced disorder of the immune system, we focused on genomic regions containing genes related to immune responses. Our results identified seven key genes (TRAF3IP2, WDR7, SWAP70, TNFRSF11A, CBFB, IGF2R, and GPR65) related to immune system process, which might play causal roles in immune-mediated responses to AMDV infection. The TRAF3IP2 gene was detected at scaffold5: 9.51-9.55 Mb by integrated analysis of $\mathrm{F}_{\mathrm{ST}}$ and $\theta \pi$ in kidney lesions group. The TRAF3IP2 gene encodes nuclear factor-kappa-B $(N F-\kappa B)$ activator 1 (Act1), known as the protein that is found in a range of immune cells such as epithelial cells, B cells, T helper (Th) cells, and neutrophils cells ${ }^{59,60}$. It has been suggested that the TRAF3IP2 gene played a significant role in the homeostasis of $\mathrm{B}$ cells ${ }^{61}$ and acted as a positive regulator in the IL17-dependent signaling pathway associated with autoimmunity and inflammatory diseases ${ }^{62}$. Similar to TRAF3IP2, the SWAP70 (scaffold36: $16.85-16.92 \mathrm{Mb}$, kidney lesions group) is also a coding gene contributing in NF- $\kappa B$ signaling pathway. This gene restricts spontaneous maturation of dendritic cells and is associated with the capacity to induce immune responses ${ }^{63}$. The role of $S W A P 70$ was also reported in c-kit receptor signaling, which was the key pathway in proliferation and differentiation of mast cells ${ }^{64}$. The TNFRSF11A gene is located on scaffold6: 13.93-13.96 Mb (kidney lesion group), and involves in autoinflammatory disorders and dysfunction of the innate immune system ${ }^{65}$. The WDR7 gene was identified on scaffold6: 18.16-18.52 Mb and shared between the results obtained in both virus clearance and kidney lesions groups, which are the two important measures associated with tolerance to AD. It was reported that WDR7 plays a regulatory role in the endoplasmic reticulum contributing to protein processing and secretion ${ }^{66}$. The efficiencies of the endoplasmic reticulum can influence immunity system e.g. the maturation process of B-cells to immunoglobulin secreting plasma cells ${ }^{67}$. The $C B F B$ was the other gene related to immune responses, which was detected on scaffold3: 6.72-6.78 Mb in viremia-350 group. The $C B F B$ gene involves in the pathway of making a protein called core binding factor subunit beta $(C B F B)$, which is known to contribute in autoimmunity and inflammation ${ }^{68,69}$. In humans, it was revealed that $C B F B$ is required for expression of the $H I V$-1-restrictive APOBEC3 gene repertoire ${ }^{70,71}$. The IGF2R gene was identified on scaffold5: 22.64-22.74 Mb in virus clearance group and it was shown to enhance the regulatory T-cell functions $\mathrm{s}^{72}$ and functions of antigen-specific regulatory B cells ${ }^{73}$. Finally, a region containing the GPR65 gene was detected on scaffold1: 27.71-27.72 Mb in survival group, which was related with the immune reactions to AMDV infection. The GPR65 gene plays a central role as the critical regulator of pro-inflammatory $\mathrm{T}$ cell (Th17) pathogenicity ${ }^{74}$. This key gene also involves in immune response by maintaining lysosome function and may have a function in activation-induced cell death or differentiation of T-cells ${ }^{75,76}$. 
Reproductive performances. It has been confirmed that AMDV infection causes reduced litter sizes and pregnancy rates, and increases abortion and mortality rates in American mink ${ }^{5}$. Our results revealed multiple genes associated with reproductive functions, which might play potential roles connected to these impacts. For instance, it was shown that FBXO5 (scaffold5: 16.61-16.62 Mb, virus clearance group) is strongly expressed in oocyte and blastocyst tisseus ${ }^{77,78}$ and is a down-regulated gene arresting the differentiation and growth of the human cumulus cell in the periovulatory period ${ }^{79}$. Furthermore, the CatSper $\beta$ gene was detected in virus clearance group on scaffold $1: 24.56-24.67 \mathrm{Mb}$ and was associated with sperm cell hyperactivation. It was shown that this gene is essential for sperm motility as well as the preparation of sperm for fertilization ${ }^{80}$. Similarly, the CATSPER4 gene (scaffold10: 17.41-17.42 Mb, survival group) is related with the male infertility ${ }^{81}$. The IGF2R gene was identified on scaffold5: 22.64-22.74 Mb in virus clearance group and it seems that the expression of this gene plays critical roles in pre-implantation of embryos ${ }^{82}$ and birth weight ${ }^{83}$. The effect of this gene was also reported for growth traits in Egyptian buffalo ${ }^{84}$. Finally, it was revealed that the GOT2 gene (scaffold3: 13.84-13.87 Mb, kidney lesions group) is expressed in placenta and can be influenced by maternal factors ${ }^{85}$. These results revealed the possible connection between potentially selected genes for response to AMDV infection and reproductive performances in American mink.

Other responses. Our results revealed several candidate genes related to heart (FHOD3, TENM4, WNT11 and NDRG4), liver (SULF2, SRSF5 and IGF2R) and kidney (SULF2 and WNT11) developments (Supplementary Dataset S2), which might be associated with the clinical signs caused by AD in these tissues ${ }^{86}$. Moreover, many candidate genes were related to response to stress (SULF2, CADPS2, NOX3, GNAO1, HSF4, AMFR, and CNOT1) and regulation of nervous system (TENM4, VSNL1, CLSTN1, RIT2, TCF4, SKOR2, and NDRG4), which might indicate the significant role of response to stimulus during the AD development (Supplementary Dataset S2).

Candidate regions shared between various phenotypic groups. In total, seven overlapped regions were observed between at least two groups of individuals, providing independent supports for the candidate regions (Table 3). The most shared selected regions were identified in the survival group (4 regions), suggesting that this group can be used as the best measure to scan the genomic signatures of selection in American mink for response to $\mathrm{AD}$. On the other hand, the lowest number of shared selected regions was revealed by viremia-350 group.

We identified ten candidate genes on the seven putatively selected regions shared between at least two groups. Four genes (SLC39A9, PLEKHD1, SRSF5 and SLC10A1) were shared among the putative positions detected on scaffold 1 by antibody titer and virus clearance groups (Table 3). The SLC39A9 gene (scaffold1: $22.80-22.86 \mathrm{Mb}$ ) encodes the zinc transporter ZIP9 protein, which regulates zinc homeostasis in the secretory pathway ${ }^{87}$. The SLC39A9 gene played an essential role in regulating the activations of Akt and Erk in B-Cell receptor signaling pathway in chicken DT40 cells ${ }^{88}$. This gene was also identified as a candidate gene involving in the molecular mechanisms underlying chikungunya virus infection in human ${ }^{89}$. The PLEKHD1 gene (scaffold1: $22.87-22.90 \mathrm{Mb}$ ) was located on the upstream of SLC39A9 gene and potentially interacted with SLC39A9 by forming chimeric genes ${ }^{90}$. The SRSF5 gene (scaffold1: 23.11-23.12 Mb) constitutes a protein which is a member of the serine/arginine (SR)-rich family of pre-mRNA splicing factors ${ }^{91}$. The SRSF5 is known as a main regulator of human immunodeficiency virus type 1 (HIV-1) mRNA splicing ${ }^{92}$ and promotes the translation of un-spliced HIV-1 RNA ${ }^{93}$. In addition, it was identified as insulin-induced protein in regenerating liver ${ }^{94}$. The SLC10A1 gene was also detected on the same region (scaffold1:23.12-23.15 Mb) and predominantly expressed on hepatic basolateral membranes ${ }^{95}$. This gene plays key roles as bile acid transporter ${ }^{96}$ as well as the receptor for hepatitis $\mathrm{B}$ and $\mathrm{D}$ virus ${ }^{97}$. It was suggested that product of this gene regulated the innate antiviral responses in liver ${ }^{98}$.

Two genes (GALNT1 and SLC39A6) were found within the putatively selected regions on scaffold2: 13.75-14.40 Mb, which were detected by both survival and antibody titer groups (Table 3). It was suggested that expression of GALNT1 gene was required for the normal heart valve development and cardiac function ${ }^{99}$. The SLC39A6 gene encodes zinc transporter ZIP6, which is essential for the function of the lymphocyte activation machinery ${ }^{100}$. Moreover, the SLC39A6 plays a functional role in preparation of monoclonal antibodies ${ }^{101}$, which could modulate anti-AMDV antibody production. Another candidate region located on scaffold 2 (23.42-24.15 Mb) was validated by the analyses of survival, viremia-350 and antibody titer groups (Table 3 ). This selected region contains RNF165, LOXHD1 and SKOR2 genes. The RNF165 and SKOR2 genes were annotated to be involved in response to stimulus. The RNF165 gene involves in epigenetic programming of T-cell phenotypes during early development in humans ${ }^{102}$. The SKOR2 gene is a member of the SKI protein family, which was known as one of the major negative regulators of the transforming growth factor- $\beta$ (TGF- $\beta$ ) signaling pathway. It was indicated that loss of TGF- $\beta$ signaling was associated with inflammation and autoimmune diseases ${ }^{103}$, which is in accordance with the fact that $\mathrm{AD}$ is a virus-induced disorder of the immune system and autoimmune disease ${ }^{1}$. Moreover, SKOR2 gene was associated with female infertility in inbred mice ${ }^{104}$. The LOXHD1 gene encodes a highly conserved protein that localizes along the plasma membrane of stereocilia in the hair cells. This gene was expressed in the mechanosensory hair cells and was related to auditory defects in mice, showing that it is required for normal hair cell function in the inner ear ${ }^{105}$. In addition, mutations within LOXHD1 were related to nonsyndromic hearing loss in humans ${ }^{106}$.

Finally, two 13.47-13.95 Mb and 18.37-19.42 Mb regions on scaffold 6 were shared between the suggestive positions detected by kidney lesions and virus clearance groups. The presence of common genes controlling these two phenotypes is reasonable since kidney lesions are caused by deposition of antibody-virus complexes ${ }^{29,107}$, which disappear when animals clear the virus. The region of 13.44-13.65 Mb contains the PHLPP1 gene, which regulates the transcription of genes involved in magnitude and duration of inflammatory signaling and innate immune responses ${ }^{108}$. It was suggested that reduced expression of PHLPP1 enhanced the antiapoptotic B-cell 
receptor signal in chronic lymphocytic leukemia B-cells ${ }^{109}$. The other suggestive selected region was detected on scaffold6: 18.37-19.42 Mb, shared between kidney lesions and virus clearance groups. This region contains the WDR7 gene that plays a role in immunity system as described in the previous section. In addition, we found the TCF4 gene located in the downstream of this region (scaffold6: 19.40-19.79 Mb), which was known as an immunoglobulin enhancer expressing predominantly in pre-B-cells ${ }^{110}$. The protein encoded by this gene (transcription factor E2-2) was identified as the critical regulator of plasmacytoid dendritic cells (PDCs) development. The PDCs provide a unique immune cell type specialized in type I interferon (IFN) secretion in response to viral nucleic acids ${ }^{111}$.

Validation by HapFLK test. Given the fact that the number of small scaffolds was high in the data set, the hapFLK tests were simply restricted to those regions detected by differentiation measures in the previous step. As expected, a larger number of candidate regions were detected by integrated measurement of differential statistics $\left(\mathrm{F}_{\mathrm{ST}}\right.$ and $\left.\theta \pi\right)$ compared to those identified by haplotype-based hapFLK test. In fact, hapFLK test can only identify selected regions with long haplotypes whereas differential methods are more sensitive to detect shorter candidate regions ${ }^{112}$. However, $50 \%$ of suggestive regions detected by hapFLK approach were also found by integrated measurement of $\mathrm{F}_{\mathrm{ST}}$ and $\theta \pi$, indicating that these positions can be considered as more reliable candidates of selective sweeps for AD in American mink. The haplotype region of scaffold8 (3.47-5.30 Mb) was detected by hapFLK tests of both kidney lesions and virus clearance groups, which was also validated by the genetic differential analyses (Table 4). Our results indicated that this region contained multiple key genes related to immune system process including MMP-9, CTSA, ELMO2, OCSTAMP and ACOT8. Interestingly, it has been shown that the $M M P-9$ gene plays a critical role in the clearance of autoantigens, autoantibodies and immune complexes in plasma. There is evidence that the lack of MMP-9 could lead to increase in the levels of immune complexes in plasma and local complement activation in spleen and kidneys ${ }^{113,114}$, suggesting that this gene can be a prominent candidate for variation in virus clearance among mink. This gene was also known to be involved in the ovarian responses to gonadotropins, sex hormones, and TGFB1 in Chicken ${ }^{115}$. The CTSA gene is expressed in primary antigen-presenting cells ${ }^{116}$ and the ELMO2 gene is essential in phagocytosis and cell migration ${ }^{117}$. It was revealed that the OCSTAMP gene suppresses M1 pro-inflammatory state by inducing a phenotypic switch in macrophage polarization ${ }^{118}$. Finally, the ACOT8 gene encodes a protein binding to the HIV-1 protein Nef and participates in Nef-mediated MHC-I downregulation and the prevention of T-cell activation ${ }^{119}$.

Another candidate region supported by all statistical approaches was detected on scaffold4: 10.51-10.84 Mb. Two genes, KLHDC10 and UBE2H, were identified in this selected region, which were associated with immune responses. It was suggested that KLHDC10 deficiency protected mice against TNF $\alpha$-induced systemic inflammation $^{120}$. The UBE2H gene encodes ubiquitin-conjugating enzyme E2 $\mathrm{H}$. The ubiquitin plays a crucial role in cellular mechanisms involving in proteins modifications to target abnormal or short-lived proteins for degradation ${ }^{121}$. In addition, deregulated ubiquitination events were related to autoimmune and inflammatory responses $^{122}$.

Finally, two regions including scaffold1: 23.04-23.18 Mb and scaffold2: 23.42-23.96 Mb were detected by hapFLK test of antibody titer group, which were also supported by the integrated analysis of $\mathrm{F}_{\mathrm{ST}}$ and $\theta \pi$ in two or more of the phenotypic groups (Table 4). These results suggested that these regions were strong candidates of selection for AD tolerant mink. Five key genes, RNF165, LOXHD1, SRSF5, SLC10A1 and SKOR2, were shared among these suggestive regions. These genes, as described in the previous sections, might involve in critical responses to AMDV infection, such as immune responses, virus-host interactions, reproductive process, liver regeneration and response to stimulus.

Although there is no study on detecting genomic signatures of selection in American mink, this approach has been used to analyze resistance against diseases in other domestic animals e.g. susceptibility to gastrointestinal nematodes in sheep ${ }^{123}$ and resistance to Marek's disease in inbred chicken lines ${ }^{124}$. In addition, recent studies indicated that pathogen-driven selection has played a strong role in human genome evolution ${ }^{125}$. For instance, strong selection signatures were identified in multiple loci in relation with human immunology ${ }^{126}$, adaptation to pathogen pressure ${ }^{127}$, innate immunity ${ }^{128}$ and histocompatibility complex (MHC) regions ${ }^{129}$. We identified numerous well-documented genes related to immune responses, reproduction, liver and kidney functions which might contribute to AD tolerance in American mink. The combination of several groups of AD-related phenotypes as well as integration of different statistical approaches were used to support the results of this study. However, owing to the lack of complete/correct genome annotation in American mink, the issue of false positive and gene missingness could not be assessed in this study. Thus, further investigations would be required to validate the role of these putatively selected regions in AD control. The collection of suggested genes in this study presents a foundation for development of molecular approaches to unveil the response of mink to AMDV infection. In addition, given the fact that the lack of chromosome-scale information restricted the analyses of this study to larger scaffolds ( $>10 \mathrm{Mb}$ ), the availability of chromosome-scale genome assembly can improve the detection of selection signatures in future studies.

The AMDV is known as a parvovirus with a high degree of variability that can infect a wide range of the Mustelidae family e.g. European mink, ferrets, Eurasian otters, stone martens, polecats and pine martens. Moreover, the occurrence of AMDV infection was reported in other small carnivores e.g. striped skunks, foxes, common genets and raccoons ${ }^{28,130}$. Accordingly, both host factors and viral strains can affect the pathogenesis of AMDV in the infected animals. Obviously, the results of this study could be extended for further research in the other susceptible species. It is suggested to take advantage of transcriptome analysis in the other related species to investigate the candidate genes revealed in the current study.

Selection for tolerant mink is certainly a feasible approach to control the AMDV infection on mink farms. Genomic selection can be applied as a useful breeding strategy to improve the economically important traits 
in the mink industry ${ }^{131}$. Detection of numerous loci in this study implies that genomic selection would be an effective approach to increase the tolerance against AMDV infection in mink. Although results of the current study confirmed that survival phenotype could provide the most effective measurement to reveal the selected regions of genome for response to AMDV infection, collecting such records on farms cannot be practical owing to high costs and technical issues. The antibody titer test is not only a practical measurement in farms, but also contributed to identify significant candidate regions in the current study, making it a suggestive phenotype to select tolerant animals in farms.

\section{Conclusion}

This study provided the first map of underlying selection signals of AD in American mink. The integrated results of genetic differentiation analyses as well as haplotype-based tests revealed numerous well-known genes related to AD phenotypes. Two strongly selected regions were detected on scaffold1: $23.04-23.18 \mathrm{Mb}$ and scaffold2: 23.42-23.96 Mb, which contained the critical genes involving in immune responses, virus-host interactions, reproductive process, liver regeneration and response to stimulus. The functional annotation supported the association of $\mathrm{AD}$ phenotypes with genomic regions detected in the current study. However, further investigation will be essential to validate the functions of these putatively selected regions for response to AMDV infection. Detection of multiple loci undergoing the selection for AMDV infection indicated that genomic selection can be applied as a feasible approach to control this disease. The survival phenotype was recognized as the best measure to reveal genomic signatures of selection for response to AMDV infection. However, the antibody titer test could be suggested as the applicable measurement to develop genomic selection for increasing $\mathrm{AD}$ tolerance in mink farms.

\section{Data availability}

The datasets generated/analyzed for this study are available from the FigShare Repository: https://doi.org/10.6084/ m9.figshare.9757784.

Received: 20 June 2020; Accepted: 21 January 2021

Published online: 03 February 2021

\section{References}

1. Bloom, M. E., Kanno, H., Mori, S. \& Wolfinbarger, J. B. Aleutian mink disease: Puzzles and paradigms. Infect. Agents Dis. 3, 279-301 (1994).

2. Porter, D. D., Larsen, A. E. \& Porter, H. G. The pathogenesis of Aleutian disease of mink. 3. Immune complex arteritis. Am. J. Pathol. 71, 331-344 (1973)

3. Valdovska, A. \& Pilmane, M. Histopathologic and immunohistochemical lesions in liver of mink infected with Aleutian disease virus. Pol. J. Vet. Sci. 14, 69-76 (2011).

4. Jensen, T. H., Chriél, M. \& Hansen, M. S. Progression of experimental chronic Aleutian mink disease virus infection. Acta Vet. Scand. 58, 35 (2016).

5. Broll, S. \& Alexandersen, S. Investigation of the pathogenesis of transplacental transmission of Aleutian mink disease parvovirus in experimentally infected mink. J. Virol. 70, 1455-1466 (1996).

6. Farid, A. H. \& Ferns, L. E. Aleutian mink disease virus infection may cause hair depigmentation. Scientifur 35, 55-59 (2011).

7. Farid, A. H., Zillig, M. L., Finley, G. G. \& Smith, G. C. Prevalence of the Aleutian mink disease virus infection in Nova Scotia, Canada. Prev. Vet. Med. 106, 332-338 (2012).

8. Prieto, A. et al. Distribution of Aleutian mink disease virus contamination in the environment of infected mink farms. Vet. Microbiol. 204, 59-63 (2017).

9. Kowalczyk, M., Horecka, B. \& Jakubczak, A. Aleutian mink disease virus in the breeding environment in Poland and its place in the global epidemiology of AMDV. Virus Res. 270, 197665 (2019).

10. Liu, D. et al. Construction and immunogenicity analysis of whole-gene mutation DNA vaccine of Aleutian mink virus isolated virulent strain. Viral Immunol. 31, 69-77 (2018).

11. An, S. H., Depauli, F. J., Wright, P. \& Ingram, D. G. Characteristics of inapparent Aleutian disease virus infection in mink. Res. Vet. Sci. 24, 200-204 (1978).

12. Cho, H. J. \& Greenfield, J. Eradication of Aleutian disease of mink by eliminating positive counterimmunoelectrophoresis test reactors. J. Clin. Microbiol. 7, 18-22 (1978).

13. Hadlow, W. J., Race, R. E. \& Kennedy, R. C. Royal pastel mink respond variously to inoculation with Aleutian disease virus of low virulence. J. Virol. 50, 38-41 (1984).

14. Aasted, B. \& Hauch, H. Studies on the progression of Aleutian disease in mink. Acta Vet. Scand. 29, 315-321 (1988).

15. Farid, A. H., Daftarian, P. M. \& Fatehi, J. Transmission dynamics of Aleutian mink disease virus on a farm under test and removal scheme. J. Vet. Sci. Med. Diag. 7, 2-10 (2018).

16. Larsen, A. E. \& Porter, D. D. Pathogenesis of aleutian disease of mink: Identification of nonpersistent infections. Infect. Immun. 11, 92-94 (1975).

17. Vitti, J. J., Grossman, S. R. \& Sabeti, P. C. Detecting natural selection in genomic data. Annu. Rev. Genet. 47, 97-120 (2013).

18. Rubin, C. J. et al. Whole-genome resequencing reveals loci under selection during chicken domestication. Nature 464, 587 (2010).

19. Rubin, C. J. et al. Strong signatures of selection in the domestic pig genome. Proc. Natl. Acad. Sci. USA 109, 19529-19536 (2012).

20. Yang, J. et al. Whole-genome sequencing of native sheep provides insights into rapid adaptations to extreme environments. Mol. Biol. Evol. 33, 2576-2592 (2016).

21. Qu, Y. et al. Ground tit genome reveals avian adaptation to living at high altitudes in the Tibetan plateau. Nat. Commun. 4, 2071 (2013).

22. Guo, J. et al. Whole-genome sequencing reveals selection signatures associated with important traits in six goat breeds. Sci. Rep. 8, 10405 (2018).

23. Liu, Z. et al. Genome-wide analysis reveals signatures of selection for important traits in domestic sheep from different ecoregions. BMC Genom. 17, 863-863 (2016).

24. Gurgul, A. et al. Genotyping-by-sequencing performance in selected livestock species. Genomics 111, 186-195 (2019).

25. Farid, A. H., Hussain, I. \& Arju, I. Detection of Aleutian mink disease virus DNA and antiviral antibodies in American mink (Neovison vison) 10 days postinoculation. J. Vet. Diagn. Investig. 27, 287-294 (2015). 
26. Farid, A. H. \& Hussain, I. Dose response of black American mink to Aleutian mink disease virus. Immun. Inflam. Dis. 8, 150-164 (2020).

27. Farid, A. H. \& Hussain, I. A comparison between intraperitoneal injection and intranasal and oral inoculation of mink with Aleutian mink disease virus. Res. Vet. Sci. 124, 85-92 (2019).

28. Farid, A. H. Aleutian mink disease virus in furbearing mammals in Nova Scotia, Canada. Acta Vet. Scand. 55, 10-10 (2013).

29. Henson, J. B., Gorham, J. R., McGuire, T. C. \& Crawford, T. B. Pathology and pathogenesis of Aleutian disease. Front. Biol. 44, 175-205 (1976).

30. Aljanabi, S. M. \& Martinez, I. Universal and rapid salt-extraction of high quality genomic DNA for PCR-based techniques. Nucleic Acids Res. 25, 4692-4693 (1997).

31. Martin, M. Cutadapt removes adapter sequences from high-throughput sequencing reads. EMBnet J. 17, 3 (2011).

32. Cai, Z. et al. The first draft reference genome of the American mink (Neovison vison). Sci. Rep. 7, 14564 (2017).

33. Li, H. \& Durbin, R. Fast and accurate short read alignment with Burrows-Wheeler transform. Bioinformatics 25, 1754-1760 (2009).

34. McKenna, A. et al. The genome analysis toolkit: A MapReduce framework for analyzing next-generation DNA sequencing data. Genome Res. 20, 1297-1303 (2010).

35. Danecek, P. et al. The variant call format and VCFtools. Bioinformatics 27, 2156-2158 (2011).

36. Weir, B. S. \& Cockerham, C. C. Estimating F-statistics for the analysis of population structure. Evolution 38, 1358-1370 (1984).

37. Nei, M. \& Li, W. H. Mathematical model for studying genetic variation in terms of restriction endonucleases. Proc. Natl. Acad. Sci. USA 76, 5269-5273 (1979).

38. Krzywinski, M. I. et al. Circos: An information aesthetic for comparative genomics. Genome Res. 19, 1639-1645 (2009).

39. Fariello, M. I., Boitard, S., Naya, H., SanCristobal, M. \& Servin, B. Detecting signatures of selection through haplotype differentiation among hierarchically structured populations. Genetics 193, 929 (2013).

40. Scheet, P. \& Stephens, M. A fast and flexible statistical model for large-scale population genotype data: Applications to inferring missing genotypes and haplotypic phase. Am. J. Hum. Genet. 78, 629-644 (2006).

41. Fariello, M. I. et al. Selection signatures in worldwide sheep populations. PLoS ONE 9, e103813 (2014).

42. Storey, J. D. \& Tibshirani, R. Statistical significance for genomewide studies. Proc. Natl. Acad. Sci. 100, 9440-9445 (2003).

43. Thomas, P. D. et al. PANTHER: A library of protein families and subfamilies indexed by function. Genome Res. 13, 2129-2141 (2003).

44. Cai, Z. et al. SNP markers associated with body size and pelt length in American mink (Neovison vison). BMC Genet. 19, 103 (2018).

45. Ma, Y. et al. Genomic analysis to identify signatures of artificial selection and loci associated with important economic traits in Duroc pigs. Genes Genom. Genet. 8, 3617 (2018).

46. Bahbahani, H. et al. Genome diversity and signatures of selection for production and performance traits in dromedary camels. Front. Genet. 10, 893 (2019).

47. Lai, F.-N. et al. Whole-genome scanning for the litter size trait associated genes and SNPs under selection in dairy goat (Capra hircus). Sci. Rep. 6, 38096-38096 (2016).

48. Ma, H. et al. Identifying selection signatures for backfat thickness in Yorkshire pigs highlights new regions affecting fat metabolism. Genes 10, 254 (2019).

49. López, M. E. et al. Multiple selection signatures in farmed Atlantic salmon adapted to different environments across hemispheres. Front. Genet. 10, 901 (2019).

50. Okada, Y. et al. Deep whole-genome sequencing reveals recent selection signatures linked to evolution and disease risk of Japanese. Nat. Commun. 9, 1631 (2018).

51. Ma, Y. et al. Properties of different selection signature statistics and a new strategy for combining them. Heredity 115, 426-436 (2015).

52. Jackson, M. K., Ellis, L. C., Morrey, J. D., Li, Z. Z. \& Barnard, D. L. Progression of Aleutian disease in natural and experimentally induced infections of mink. Am. J. Vet. Res. 57, 1753-1758 (1996).

53. Jensen, T. H., Hammer, A. S. \& Chriél, M. Monitoring chronic infection with a field strain of Aleutian mink disease virus. Vet. Microbiol. 168, 420-427 (2014).

54. Jensen, T. H., Christensen, L. S., Chriél, M., Uttenthal, Å. \& Hammer, A. S. Implementation and validation of a sensitive PCR detection method in the eradication campaign against Aleutian mink disease virus. J. Virol. Methods 171, 81-85 (2011).

55. Kanno, H., Wolfinbarger, J. B. \& Bloom, M. E. Aleutian mink disease parvovirus infection of mink macrophages and human macrophage cell line U937: demonstration of antibody-dependent enhancement of infection. J. Virol. 67, 7017-7024 (1993).

56. Canuti, M. et al. Driving forces behind the evolution of the Aleutian mink disease parvovirus in the context of intensive farming. Virus Evol. 2, vew004 (2016).

57. Leader, R. W., Wagner, B. M., Henson, J. B. \& Gorham, J. R. Structural and histochemical observations of liver and kidney in Aleutian disease of mink. Am. J. Pathol. 43, 33-53 (1963).

58. De, A. Wnt/Ca2+ signaling pathway: A brief overview. Acta Biochem. Biophys. Sin. 43, 745-756 (2011).

59. Ambarus, C., Yeremenko, N., Tak, P. P. \& Baeten, D. Pathogenesis of spondyloarthritis: Autoimmune or autoinflammatory?. Curr. Opin. Rheumatol. 24, 351-358 (2012).

60. Doyle, M. S., Collins, E. S., FitzGerald, O. M. \& Pennington, S. R. New insight into the functions of the interleukin-17 receptor adaptor protein Actl in psoriatic arthritis. Arthr. Res. Ther. 14, 226 (2012).

61. Qian, Y. et al. Act1, a negative regulator in CD40- and BAFF-mediated B cell survival. Immunity 21, 575-587 (2004).

62. Qian, Y. et al. The adaptor Act1 is required for interleukin 17-dependent signaling associated with autoimmune and inflammatory disease. Nat. Immunol. 8, 247-256 (2007).

63. Ocaña-Morgner, C., Götz, A., Wahren, C. \& Jessberger, R. SWAP-70 restricts spontaneous maturation of dendritic cells. J. Immunol. 190, 5545-5558 (2013).

64. Sivalenka, R. R. \& Jessberger, R. SWAP-70 regulates c-kit-Induced Mast cell activation, cell-cell adhesion, and migration. Mol. Cell Biol. 24, 10277-10288 (2004).

65. Jéru, I. et al. Brief report: involvement of TNFRSF11A molecular defects in autoinflammatory disorders. Arthr. Rheumatol. 66, 2621-2627 (2014).

66. Cheung, V. G. et al. Polymorphic cis- and trans-regulation of human gene expression. PLOS Biol. 8, e1000480 (2010).

67. Todd, D. J., Lee, A.-H. \& Glimcher, L. H. The endoplasmic reticulum stress response in immunity and autoimmunity. Nat. Rev. Immunol. 8, 663-674 (2008).

68. Kahlon, S., Shreibman, D., Unger, T., Ben-Yehuda, D. \& Elias, S. The oncogenic fusion protein CBFB-SMMHC downregulates CD48 to evade NK cell recognition. Blood Cancer J. 8, 48 (2018).

69. Speck, N. A. et al. Core-binding factor: A central player in hematopoiesis and leukemia. Cancer Res. 59, 1789s-1793s (1999).

70. Anderson, B. D. \& Harris, R. S. Transcriptional regulation of APOBEC 3 antiviral immunity through the CBF- $\beta /$ RUNX axis. Sci. Adv. 1, e1500296-e1500296 (2015).

71. Jäger, S. et al. Vif hijacks CBF- $\beta$ to degrade APOBEC3G and promote HIV-1 infection. Nature 481, 371-375 (2012).

72. Yang, G. et al. Insulin-like growth factor 2 enhances regulatory T-cell functions and suppresses food allergy in an experimental model. J. Allergy Clin. Immunol. 133, 1702-1708.e1705 (2014). 
73. Geng, X. R. et al. Insulin-like growth factor-2 enhances functions of antigen (Ag)-specific regulatory B cells. J. Biol. Chem. 289, 17941-17950 (2014).

74. Hernandez, J. B. GPR65, a critical regulator of Th17 cell pathogenicity, is regulated by the CRTC2/CREB pathway. J. Immunol. 200, $110.112(2018)$.

75. Lassen, K. G. et al. Genetic coding variant in GPR65 alters lysosomal PH and links lysosomal dysfunction with colitis risk. Immunity 44, 1392-1405 (2016).

76. Kyaw, H. et al. Cloning, characterization, and mapping of human homolog of mouse T-cell death-associated gene. DNA Cell Biol. 17, 493-500 (1998).

77. Assou, S. et al. The human cumulus-oocyte complex gene-expression profile. Hum. Reprod. 21, 1705-1719 (2006).

78. Shaw, L., Sneddon, S. F., Zeef, L., Kimber, S. J. \& Brison, D. R. Global gene expression profiling of individual human oocytes and embryos demonstrates heterogeneity in early development. PLoS ONE 8, e64192 (2013).

79. Yerushalmi, G. M. et al. Characterization of the human cumulus cell transcriptome during final follicular maturation and ovulation. Mol. Hum. Reprod. 20, 719-735 (2014).

80. Liu, J., Xia, J., Cho, K. H., Clapham, D. E. \& Ren, D. CatSper $\beta$, a novel transmembrane protein in the CatSper channel complex. J. Biol. Chem. 282, 18945-18952 (2007).

81. Carkci, S. et al. Ion channel gene expressions in infertile men: A case-control study. Int. J. Reprod. Biomed. 15, 749-756 (2017).

82. Latham, K. E., Doherty, A. S., Scott, C. D. \& Schultz, R. M. IGF2R and IGF2 gene expression in androgenetic, gynogenetic, and parthenogenetic preimplantation mouse embryos: absence of regulation by genomic imprinting. Genes Dev. 8, 290-299 (1994).

83. Demetriou, C. et al. Paternally expressed, imprinted insulin-like growth factor- 2 in chorionic villi correlates significantly with birth weight. PLoS ONE 9, e85454 (2014).

84. El-Magd, M. A., Abo-Al-Ela, H. G., El-Nahas, A., Saleh, A. A. \& Mansour, A. A. Effects of a novel SNP of IGF2R gene on growth traits and expression rate of IGF2R and IGF2 genes in gluteus medius muscle of Egyptian buffalo. Gene 540, 133-139 (2014).

85. Day, P. E. et al. Maternal factors are associated with the expression of placental genes involved in amino acid metabolism and transport. PLoS ONE 10, e0143653 (2015).

86. Hadlow, W. J., Race, R. E. \& Kennedy, R. C. Comparative pathogenicity of four strains of Aleutian disease virus for pastel and sapphire mink. Infect. Immun. 41, 1016-1023 (1983).

87. Matsuura, W. et al. SLC39A9 (ZIP9) regulates zinc homeostasis in the secretory pathway: characterization of the ZIP subfamily I protein in vertebrate cells. Biosci. Biotechnol. Biochem. 73, 1142-1148 (2009).

88. Taniguchi, M. et al. Essential role of the zinc transporter ZIP9/SLC39A9 in regulating the activations of Akt and Erk in B-cell receptor signaling pathway in DT40 cells. PLoS ONE 8, e58022 (2013).

89. Tanaka, A. et al. Genome-wide screening uncovers the significance of $\mathrm{N}$-sulfation of heparan sulfate as a host cell factor for chikungunya virus infection. J. Virol. 91, e00432-e1417 (2017).

90. Rippey, C. et al. Formation of chimeric genes by copy-number variation as a mutational mechanism in schizophrenia. Am. J. Hum. Genet. 93, 697-710 (2013).

91. Breig, O. \& Baklouti, F. Proteasome-mediated proteolysis of SRSF5 splicing factor intriguingly co-occurs with SRSF5 mRNA upregulation during late erythroid differentiation. PLOS ONE 8, e59137 (2013).

92. Caputi, M., Freund, M., Kammler, S., Asang, C. \& Schaal, H. A bidirectional SF2/ASF- and SRp40-dependent splicing enhancer regulates human immunodeficiency virus type $1 \mathrm{rev}$, env, vpu, and nef gene expression. J. Virol. 78, 6517-6526 (2004).

93. Swanson, C. M., Sherer, N. M. \& Malim, M. H. SRp40 and SRp55 promote the translation of unspliced human immunodeficiency virus type 1 RNA. J. Virol. 84, 6748-6759 (2010).

94. Diamond, R. H. et al. Novel delayed-early and highly insulin-induced growth response genes. Identification of HRS, a potential regulator of alternative pre-mRNA splicing. J. Biol. Chem. 268, 15185-15192 (1993).

95. Zhou, X. et al. Structural basis of the alternating-access mechanism in a bile acid transporter. Nature 505, 569-573 (2014).

96. Claro da Silva, T., Polli, J. E. \& Swaan, P. W. The solute carrier family 10 (SLC10): Beyond bile acid transport. Mol. Asp. Med. 34, 252-269 (2013).

97. Yan, H. et al. Sodium taurocholate cotransporting polypeptide is a functional receptor for human hepatitis B and D virus. eLife 1, e00049 (2012)

98. Verrier, E. R. et al. Solute carrier NTCP regulates innate antiviral immune responses targeting hepatitis c virus infection of hepatocytes. Cell Rep. 17, 1357-1368 (2016).

99. Tian, E. et al. Galnt1 is required for normal heart valve development and cardiac function. PLoS ONE 10, e0115861 (2015).

100. Colomar-Carando, N. et al. Zip6 transporter is an essential component of the lymphocyte activation machinery. J. Immunol. 202, 441-450 (2019).

101. Bagheri, S. et al. Recombinant expression of Zinc transporter SLC39A6 and its functional antibody production. Monoclon. Antib. Immunodiagn. Immunother. 38, 70-74 (2019).

102. Martino, D., Maksimovic, J., Joo, J. H., Prescott, S. L. \& Saffery, R. Genome-scale profiling reveals a subset of genes regulated by DNA methylation that program somatic T-cell phenotypes in humans. Genes Immun. 13, 388-398 (2012).

103. Tecalco-Cruz, A. C., Ríos-López, D. G., Vázquez-Victorio, G., Rosales-Alvarez, R. E. \& Macías-Silva, M. Transcriptional cofactors Ski and SnoN are major regulators of the TGF- $\beta /$ Smad signaling pathway in health and disease. Signal Transduc. Tar 3, 15 (2018).

104. Liu, J. L., Wang, T. S. \& Zhao, M. Genome-wide association mapping for female infertility in inbred mice. Genes Genom. Genet. 6, 2929-2935 (2016)

105. Grillet, N. et al. Mutations in LOXHD1, an evolutionarily conserved stereociliary protein, disrupt hair cell function in mice and cause progressive hearing loss in humans. Am. J. Hum. Genet. 85, 328-337 (2009).

106. Shen, N., Wang, T., Li, D., Liu, A. \& Lu, Y. Whole-exome sequencing identifies a novel missense variant within LOXHD1 causing rare hearing loss in a Chinese family. BMC Med. Genet. 20, 30 (2019).

107. Mori, S. et al. Interstitial nephritis in Aleutian mink disease. Possible role of cell-mediated immunity against virus-infected tubular epithelial cells. Am. J. Pathol. 144, 1326-1333 (1994).

108. Katsenelson, K. C. et al. PHLPP1 counter-regulates STAT1-mediated inflammatory signaling. eLife 8, e48609 (2019).

109. Suljagic, M. et al. Reduced expression of the tumor suppressor PHLPP1 enhances the antiapoptotic B-cell receptor signal in chronic lymphocytic leukemia B-cells. Leukemia 24, 2063-2071 (2010).

110. Reizis, B. Regulation of plasmacytoid dendritic cell development. Curr. Opin. Immunol. 22, 206-211 (2010).

111. Cisse, B. et al. Transcription factor E2-2 is an essential and specific regulator of plasmacytoid dendritic cell development. Cell 135, 37-48 (2008).

112. Yurchenko, A. A. et al. Scans for signatures of selection in Russian cattle breed genomes reveal new candidate genes for environmental adaptation and acclimation. Sci. Rep. 8, 12984 (2018).

113. Ugarte-Berzal, E. et al. MMP-9/Gelatinase B degrades immune complexes in systemic lupus erythematosus. Front. Immunol. 10, 538 (2019).

114. Teles, R. M. B. et al. High matrix metalloproteinase production correlates with immune activation and leukocyte migration in leprosy reactional lesions. Infect. Immun. 78, 1012-1021 (2010).

115. Zhu, G. et al. Expression and regulation of $M M P 1, M M P 3$, and $M M P 9$ in the chicken ovary in response to gonadotropins, sex hormones, and TGFB11. Biol. Reprod. 90, 57-1 (2014). 
116. Reich, M. et al. Cathepsin A is expressed in primary human antigen-presenting cells. Immunol. Lett. 128, 143-147 (2010).

117. Gumienny, T. L. et al. CED-12/ELMO, a novel member of the CrkII/Dock180/Rac pathway, is required for phagocytosis and cell migration. Cell 107, 27-41 (2001).

118. Yuan, H. et al. Osteoclast stimulatory transmembrane protein induces a phenotypic switch in macrophage polarization suppressing an M1 pro-inflammatory state. Acta Biochem. Biophys. Sin. 49, 935-944 (2017).

119. Liu, L. X. et al. Binding of HIV-1 Nef to a novel thioesterase enzyme correlates with Nef-mediated CD4 down-regulation. J. Biol. Chem. 272, 13779-13785 (1997).

120. Yamaguchi, N., Sekine, S., Naguro, I., Sekine, Y. \& Ichijo, H. KLHDC10 deficiency protects mice against TNFa-induced systemic inflammation. PLoS ONE 11, e0163118 (2016).

121. Lecker, S. H., Goldberg, A. L. \& Mitch, W. E. Protein degradation by the ubiquitin-proteasome pathway in normal and disease states. J. Am. Soc. Nephrol. 17, 1807-1819 (2006).

122. Hu, H. \& Sun, S.-C. Ubiquitin signaling in immune responses. Cell Res. 26, 457-483 (2016).

123. McRae, K. M., McEwan, J. C., Dodds, K. G. \& Gemmell, N. J. Signatures of selection in sheep bred for resistance or susceptibility to gastrointestinal nematodes. BMC Genom. 15, 637 (2014).

124. Xu, L. et al. Genetic assessment of inbred chicken lines indicates genomic signatures of resistance to Marek's disease. J. Anim. Sci. Biotechnol. 9, 65 (2018).

125. Cagliani, R. \& Sironi, M. Pathogen-driven selection in the human genome. Int. J. Evol. Biol. 2013, 204240 (2013).

126. Barreiro, L. B. \& Quintana-Murci, L. From evolutionary genetics to human immunology: How selection shapes host defence genes. Nat. Rev. Genet. 11, 17-30 (2010).

127. Siddle, K. J. \& Quintana-Murci, L. The Red Queen's long race: Human adaptation to pathogen pressure. Curr. Opin. Genet. Dev. 29, 31-38 (2014).

128. Deschamps, M. et al. Genomic signatures of selective pressures and introgression from Archaic Hominins at human innate immunity genes. Am. J. Hum. Genet. 98, 5-21 (2016).

129. Walter, K. et al. The UK10K project identifies rare variants in health and disease. Nature 526, 82-90 (2015).

130. Knuuttila, A. et al. Aleutian mink disease virus in free-ranging mustelids in Finland-A cross-sectional epidemiological and phylogenetic study. J. Gen. Virol. 96, 1423-1435 (2015).

131. Karimi, K., Sargolzaei, M., Plastow, G. S., Wang, Z. \& Miar, Y. Opportunities for genomic selection in American mink: A simulation study. PLoS ONE 14, e0213873 (2019).

\section{Acknowledgements}

Assistance of Irshad Hussain and Irin Arju for animal inoculation and sampling, Jeffrey Gunn and Donald Gunn for animal management and helping with phenotypic data collection, and Priyanka Rupasinghe and Laura Butler for laboratory work, is greatly acknowledged. The phenotypic data collection was possible through grants provided to AHF by the mink industry organizations (Canada Mink Breeders Association, Nova Scotia Mink Breeders Association, Fur Farmers of Canada Marketing Association, American Legend Cooperatives, North American Fur Auctions, American Mink Council, American Mink Farmers Research Foundation), Agriculture and Agri-Food Canada through the CARD Councils of Ontario, British Columbia and Nova Scotia (Agri-Futures NS), Atlantic Innovation Fund of Atlantic Opportunity Agency, and the Technology Development Program of the Nova Scotia Department of Agriculture. Laboratory analyses were performed by grants to AHF and SM by the Canada Mink Breeders Association and Agriculture and Agri-Food Canada through the Canadian Agricultural Adaptation Program of Nova Scotia (Agri-Futures Nova Scotia). Computational and genomic analyses were performed using the grants funded to YM (Mitacs Accelerate Award, NSERC Discovery Grant, Nova Scotia Mink Breeders Association, and Compute Canada-Resources for Research Group).

\section{Author contributions}

K.K. and Y.M. designed and carried out the computational and genomic analysis and interpreted the data. A.H.F. collected the phenotypic data, and A.H.F. and S.M. carried out the laboratory analyses. K.K. wrote the manuscript, and all co-authors reviewed the manuscript and approved the final draft.

\section{Competing interests}

The authors declare no competing interests.

\section{Additional information}

Supplementary Information The online version contains supplementary material available at https://doi. org/10.1038/s41598-021-82522-8.

Correspondence and requests for materials should be addressed to Y.M.

Reprints and permissions information is available at www.nature.com/reprints.

Publisher's note Springer Nature remains neutral with regard to jurisdictional claims in published maps and institutional affiliations.

Open Access This article is licensed under a Creative Commons Attribution 4.0 International License, which permits use, sharing, adaptation, distribution and reproduction in any medium or format, as long as you give appropriate credit to the original author(s) and the source, provide a link to the Creative Commons licence, and indicate if changes were made. The images or other third party material in this article are included in the article's Creative Commons licence, unless indicated otherwise in a credit line to the material. If material is not included in the article's Creative Commons licence and your intended use is not permitted by statutory regulation or exceeds the permitted use, you will need to obtain permission directly from the copyright holder. To view a copy of this licence, visit http://creativecommons.org/licenses/by/4.0/.

(C) The Author(s) 2021 